\title{
Sonographic Measurement of the Thickness of Subcutaneous Tissue and Hepatic Echo-Intensity Attenuation Rate in Non-alcoholic Fatty Liver Disease
}

Abdul Sattar Arif Khammas,2*, Hasyma Abu Hassan¹, Sarah Qahtan Mohammed Salih³, Hayati Kadir Shahar', Ramlah Mohamed Ibrahim¹,5, Nurul Nadiah Mohamed Nasir ${ }^{1,5}$, Rozi Mahmud ${ }^{6}$

${ }^{1}$ Department of Imaging, Faculty of Medicine and Health Sciences, Universiti Putra

Malaysia, Serdang, Selangor, Malaysia

${ }^{2}$ Department of Radiological Techniques, College of Health and Medical Technology, Middle Technical University, Baghdad, Iraq

${ }^{3}$ Computer Center, College of Health and Medical Technology, Middle Technical

University, Baghdad, Iraq

${ }^{4}$ Department of Public Health, Faculty of Medicine and Health Sciences, Universiti Putra Malaysia, Serdang, Selangor, Malaysia

${ }^{5}$ Department of Nutrition and Dietetics, Faculty of Medicine and Health Sciences, Universiti Putra Malaysia, Serdang, Selangor, Malaysia ${ }^{6}$ Cancer Resource and Education Centre (CaRE), Faculty of Medicine and Health Sciences, Universiti Putra Malaysia, Serdang, Selangor, Malaysia

*Corresponding author's email: abdulsattar.arif@mtu.edu.iq

Received: 24 September 2019

Accepted: 16 January 2020

Keywords:

subcutaneous tissue thickness,

hepatic echo-intensity attenuation rate, non-alcoholic fatty liver disease, sonography

\section{ABSTRACT}

The most common cause of the chronic liver disease is non-alcoholic fatty liver disease (NAFLD). This study was designed to compare a mean subcutaneous tissue thickness (SCTT) and hepatic echo-intensity attenuation rate (HEIAR) among NAFLD grades. Sonography was carried out on 628 consecutive subjects. The distance between the skin surface and the liver capsule was measured and was labelled the SCTT. Also, the ultrasound of HEIAR was retrospectively quantified on an image archiving. HEIAR was calculated as the difference between mean intensity of echo for two regions of interest (ROIs) in near- and far-fields divided by the distance between these two ROls multiply by frequency of the probe. Of the 628, 235 subjects were diagnosed with NAFLD. The age range was 45 75 years with mean $54.5 \pm 6.7$ years. There was a significant difference of mean SCTT among NAFLD grades $(p<0.001), 65.4 \%$ of subjects with SCTT measured $\geq 2.1 \mathrm{~cm}$ had NAFLD versus $34.6 \%$ of subjects had no NAFLD. Similarly, the differences of mean HEIAR among NAFLD grades were reported to be statistically significant $(p<0.001)$. All of the subjects with HEIAR of 1 . $7 \mathrm{~dB} / \mathrm{cm} \mathrm{MHz}$ and over had NAFLD. HEIAR is a useful indicator for non-invasive quantitative assessment of NAFLD where sonographically measured HEIAR equal to or over than $1.7 \mathrm{~dB} /$ cm MHz makes identifying NAFLD is probably (sensitivity is $\mathbf{5 9 \%}$ and specificity is $\mathbf{8 9} \%$ ). HEIAR is a useful indicator for non-invasive quantitative assessment of NAFLD. 


\section{INTRODUCTION}

Non-alcoholic fatty liver disease (NAFLD) is the most common cause of chronic liver disease worldwide ${ }^{1}$. It is also the most common cause for the chronic elevation of the liver enzymes in United States nowadays ${ }^{2}$. Histologically, NAFLD is defined as excessive fat accumulation in the liver tissues over $5 \%$ of the wet liver weight due to causes other than alcohol intake ${ }^{3,4}$. Dyslipidaemia is the most common condition that can lead to fatty liver disease. An abnormal circulating lipoprotein concentration has reflected the disturbances in the homeostasis of the major lipid components of TG, lipoproteins, TC, and cholesterol esters. Lipids conveyed in the blood plasma are composed of lipoprotein complexes. After eating, fat-food and TC are deposited into the intestine cells and then connected with newly resultant chylomicrons. With energy increases, the glucose is transformed into fatty acids. The latter is further utilized to build up TG. TG is stored in the form of fat droplets in the hepatocytes or merged with VLDL and then excreted into the bloodstream. In this manner, the TG content of these molecules is piecemeal decreased by the action of the lipoprotein lipase that leads to LDL with comparative increases in the cholesterol content. LDL is transported and deposited into the liver by linking the LDL to the LDL receptor. Hence, excess deposition of TG in hepatocytes is considered the hall-mark of NAFLD ${ }^{5}$. It roughly affects $20-40 \%$ of Western population 6 . In Asia, it was initially uncommon but with the increase in obesity and diabetes mellitus $(D M)^{7}$, it would also be on the increase with affecting $12-37 \%$ of general population ${ }^{8-15}$. NAFLD encompasses a wide spectrum of liver diseases ranging from simple steatosis to nonalcoholic steatohepatitis (NASH) that leads to fibrosis, cirrhosis and eventually hepatocellular carcinoma $(\mathrm{HCC})^{16}$. Nevertheless, NAFLD is still considered as a benign disease unless it develops into steatohepatitis and fibrosis ${ }^{17}$. Its association with insulin resistance and obesity has been well reported where a previous study showed that NAFLD was strongly correlated with hypertriglyceridaemia, type $2 \mathrm{DM}$ and obesity ${ }^{18}$. Furthermore, fatty liver changes are likely to be as the hepatic manifestation of the metabolic syndrome ${ }^{13}$. Interestingly, Targher, Marra \& Marchesini G (2008) $)^{19}$ indicated that NAFLD is not only a risk factor for chronic liver disease but it is also considered an independent risk factor for developing cardiovascular disease (CVD).

Currently, ultrasound is widely used for detection NAFLD because it is a noninvasive machine, safe (with no radiation hazard), available and less expensive than other radiological modalities such as magnetic resonance imaging and computed tomography ${ }^{20}$. There are sonographic features could indicate to fatty liver changes, two of the idealistic features are an increased hepatic echo-intensity attenuation rate (HEIAR) of the liver parenchyma and also increased subcutaneous tissue thickness (SCTT). Therefore, the present study was designed to define a cut-off value of the hepatic echointensity attenuation rate above which NAFLD is likely. We also sought to compare the SCTT in subjects with and without NAFLD.

\section{MATERIALS AND METHODS}

A prospective cross-sectional study was carried out among Malaysian adults with age ranges between 45 to 75 years who underwent screening programme for the period from 15 August 2015 until 15 January 2016 at Golden Horses Health Sanctuary (GHHS) located in Seri Kembangan, Klang Valley, Malaysia. Ethical from Ethics Committee for Research Involving Human Subject (JKEUPM) with number UPM/TNCPI/RMC/1.4.18.1(JKEUPM)/ F2 was obtained prior to conducting of this study. Each respondent was informed about the study verbally and in writing according to Good Clinical Practice, after that, informed consent was obtained from all respondents before the commencement of this study. 
Sonographic Measurement of the Thickness of Subcutaneous Tissue and Hepatic Echo-Intensity Attenuation Rate in Non-alcoholic Fatty Liver Disease

Males who have drunk alcohol over $140 \mathrm{~g} /$ week and females over $70 \mathrm{~g} /$ week, were hepatitis B or C viruses carrier, previous liver insults or surgery, pregnancies, taken corticosteroid or lipid-lowering medications were excluded from the study. The subjects were randomly selected using a systematic sampling method. Liver ultrasound was performed on 628 consecutive subjects by a single radiologist with experience over 10 years using Philips (HD 15) medium-range machine with a convex-array probe (3.5 MHz). A standard approach has been used in image acquisition. The subjects have been recommended not to eat any food by mouth for 8 hours prior to the examination; if the fluid is needed, only plain water should be taken. The subject lied in the supine position with his/her right arm elevated above the head. The coupling agent (gel) was applied to the right upper abdomen. The radiologist started the examination with a longitudinal scan. Afterwards, the transverse scan and intercostal scan along the mid-axillary line were achieved. The distance between the skin surface and the liver capsule was measured and labelled as the SCTT. Therefore, an attempt was made to reduce compression of subcutaneous tissue by the probe.
The normal liver parenchyma is homogenous in echotexture with reflectively (echogenicity) equal to or slightly greater than that of the renal cortex and spleen. Therefore, fatty liver changes were qualitatively assessed based on the increase in reflectively of liver parenchyma compared to the right renal cortex and spleen ${ }^{21,22}$. The determination cutoff value of HEIAR to define fatty liver changes was based on the mean of the hepatic echointensity attenuation rate among NAFLD grade I. NAFLD grades were also qualitatively assessed based on reflectively of intrahepatic vessels walls and diaphragm (Figure 1). When there is no increased in reflectively of the liver parenchyma compared to the renal cortex or spleen, it is described as grade 0 or normal. When there is slightly increased in reflectively of the liver parenchyma, it is described as grade I or mild. When there is increased in reflectively of the liver parenchyma with loss visualization of portal vascular walls, it is described as grade II or moderate. When there is markedly increased in reflectively of the liver parenchyma with poor or no visualization of the posterior portion of the diaphragm, it is described as grade III or severe ${ }^{22-25}$. 

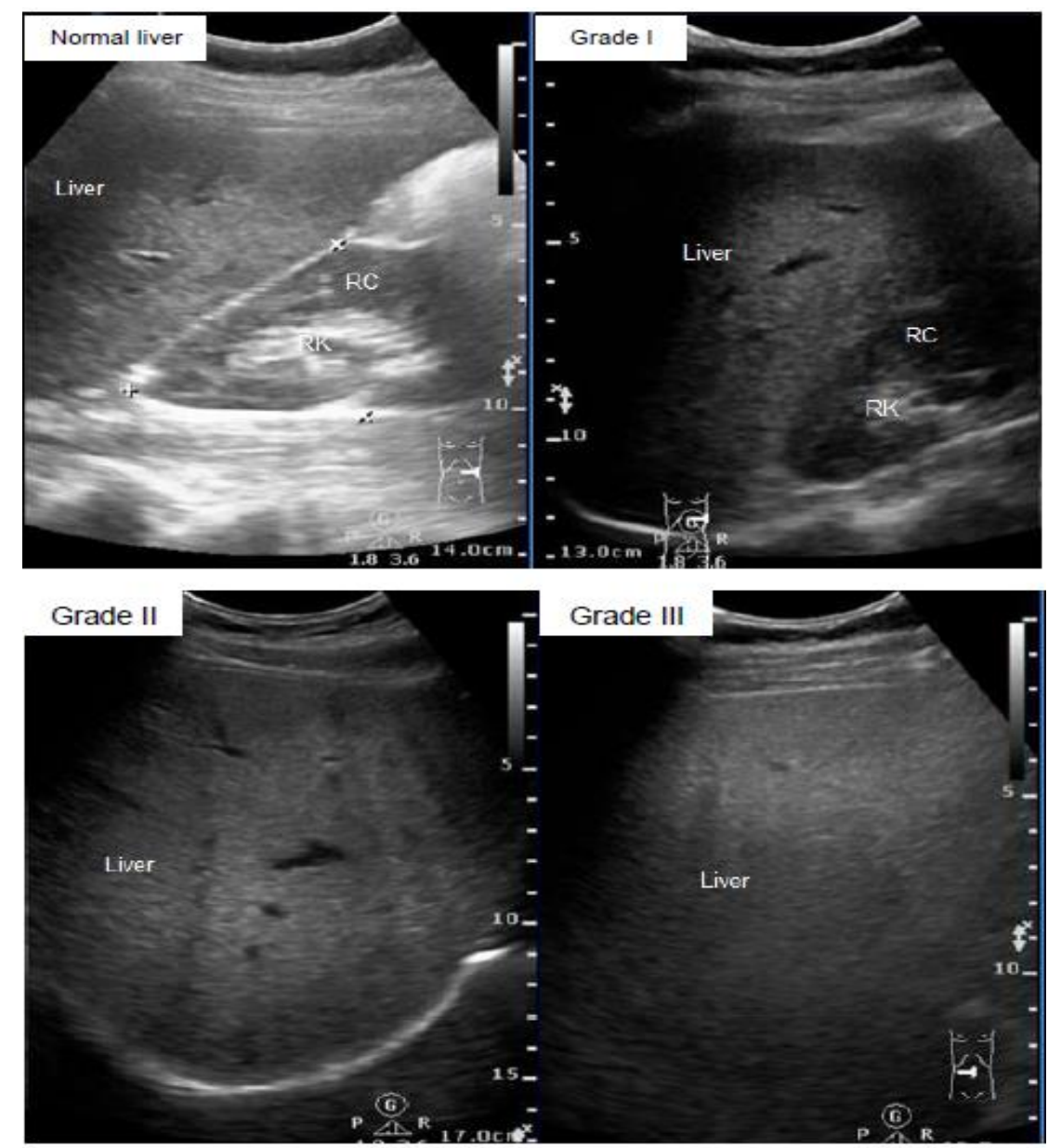

Figure 1 Various grades of diffuse fatty liver disease as compared with the normal liver (the pictures were taken from the study population)

In meanwhile, the ultrasound HEIAR was retrospectively quantified on an image archiving. On the liver image, the observer selected two ROls of $5 \mathrm{~mm}$ square in the liver homogenous area along the ultrasound beam in the near-field (depth $3-5 \mathrm{~cm}$ ) and far-field of the liver, respectively, avoiding vessels and artefacts. The distance between the two ROls were also measured (Figure 2).

HEIAR was calculated according to the equation below:

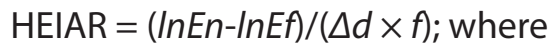

$\operatorname{InEn}$ and $\operatorname{In} E f$ are the mean intensity of echo in near-field and far-field of the liver, respectively. $\Delta d$ is the distance between two liver ROls.

$f$ is the ultrasound frequency of transducer and was used 3.5 $\mathrm{MHz}$ in this study. 
Sonographic Measurement of the Thickness of Subcutaneous Tissue and Hepatic Echo-Intensity Attenuation Rate in Non-alcoholic Fatty Liver Disease

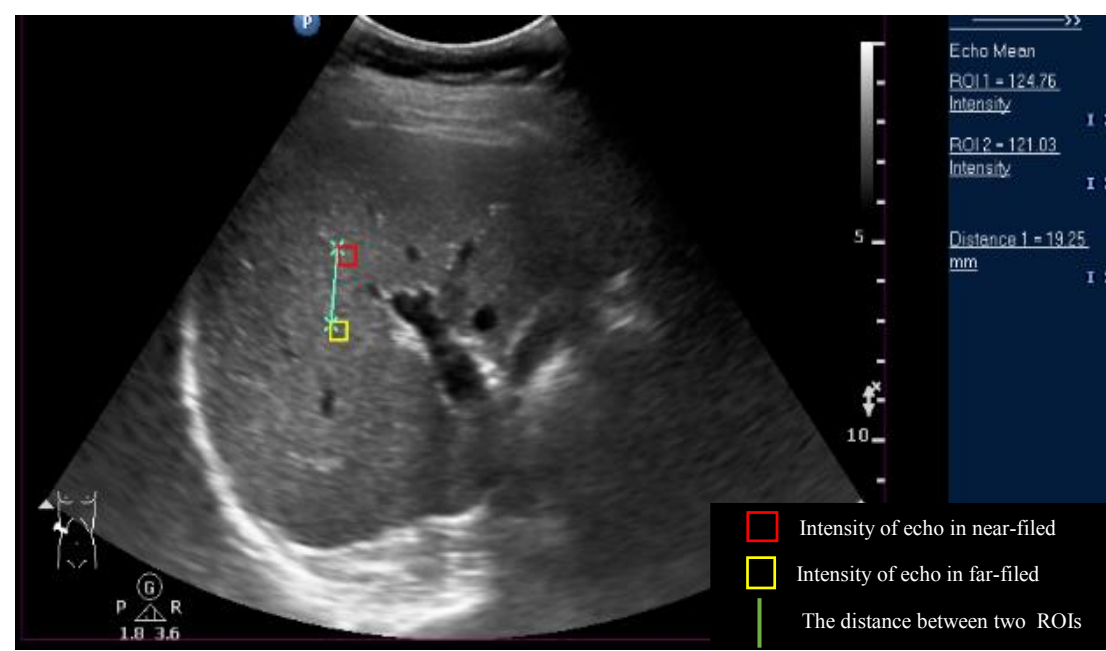

Figure 2 Hepatic echo-intensity attenuation rate on ultrasound. Image shows the liver intercostal view with the drawing of two ROIs in near-field and far-field at the homogenous area in the liver parenchyma. The distance was measured from the top corner of the near-field ROI into the top corner of far-field ROI

\section{Statistical Analysis}

Data analysis was performed using Statistical Package for Social Science (SPSS) program version 22.0. First, descriptive analysis was carried out to calculate the percentages of each factor among the study population. Chisquare test was performed to determine the association between categorical variables. In addition, sensitivity and specificity were calculated based on crosstab from the descriptive statistic. Analysis of variance (ANOVA) was achieved to compare a mean among more than three groups with normally distributed data. A $p$-value of $<0.05$ was considered statistically significant.

\section{RESULTS}

Of the 628 consecutive subjects with a mean age of $54.5 \pm 6.7$ years who underwent a screening programme and met the inclusion criteria in this study. Table 1 shows characteristics of the study population based on the distribution of age, gender, ethnicity, presence of disease and its grades. Of 235 (37.4\%) subjects diagnosed with NAFLD and $393(62.6 \%)$ subjects were not found to have NAFLD. Of those with NAFLD, there were $9.6 \%$ of subjects with a mild grade of fatty liver, $23.7 \%$ with a moderate grade and $4.1 \%$ with severe grade. The females had a higher percentage than males in our study population and overwhelming Chinese in race reflecting the urban population from which is derived from. 
Table 1 Basic socio-demographic characteristics of study population $(n=628)$

\begin{tabular}{|lcc|}
\hline Variables & $\boldsymbol{n}(\%)$ & mean \pm SD \\
\hline Age & - & $54.54 \pm 6.69$ \\
Gender & $302(48.1)$ & - \\
Male & $326(51.9)$ & - \\
Female & & - \\
Race & $92(14.6)$ & - \\
Malay & $518(82.5)$ & - \\
Chinese & $18(2.9)$ & - \\
Indian & & - \\
NAFLD & $235(37.4)$ & - \\
Yes & $393(62.6)$ & - \\
No & & - \\
NAFLD grades & $60(9.6)$ & - \\
Grade I (mild) & $149(23.7)$ & \\
Grade II (moderate) & $26(4.1)$ & \\
Grade III (severe) & & \\
\hline
\end{tabular}

\section{NAFLD: Non-alcoholic Fatty Liver Disease}

Based on one way ANOVA test, Table 2 shows the differences of means SCTT and HEIAR among NAFLD grades. The mean SCTT was $1.8 \pm 0.4 \mathrm{~cm}$ in grade 0 (normal), $2.1 \pm 0.5 \mathrm{~cm}$ in grade I (mild), $2.2 \pm 0.5 \mathrm{~cm}$ in grade II (moderate) and $2.6 \pm 0.7 \mathrm{~cm}$ in grade III (severe). This indicates that differences in mean SCTT among NAFLD grades were noted to be significant $(p<0.001)$. According to HEIAR, the results also revealed that mean HEIAR was $0.8 \pm 0.7 \mathrm{~cm}$ in grade 0 (normal), $1.7 \pm 1.3$ $\mathrm{cm}$ in grade I, $2.2 \pm 1.3$ in grade II and $3.0 \pm 1.5 \mathrm{~cm}$ in grade III. This indicates that there are significant differences in mean HEIAR among NAFLD grades $(p<0.001)$

Table 2 Differences of means HEIAR and SCTT among NAFLD grades $(n=628)$

\begin{tabular}{|c|c|c|c|c|c|c|}
\hline \multirow[t]{2}{*}{ variables } & \multicolumn{4}{|c|}{ NAFLD grades } & \multirow{2}{*}{$\begin{array}{c}\text { F-statistics } \\
\text { (df) }\end{array}$} & \multirow[b]{2}{*}{$p$-value } \\
\hline & $\begin{array}{l}\text { Grade } 0 \text { ( } n \\
=393)\end{array}$ & $\begin{array}{l}\text { Grade I ( } n \\
\quad=60)\end{array}$ & $\begin{array}{l}\text { Grade II ( } n \\
=149)\end{array}$ & $\begin{array}{l}\text { Grade III ( } n \\
\quad=26)\end{array}$ & & \\
\hline $\begin{array}{l}\text { HEIAR (dB/ } \\
\mathrm{cm} \cdot \mathrm{MHz})\end{array}$ & $0.8 \pm 0.7$ & $1.7 \pm 1.3$ & $2.2 \pm 1.3$ & $3.0 \pm 1.5$ & $9.277(2)$ & $<0.001$ \\
\hline $\mathrm{SCTT}(\mathrm{cm})$ & $1.8 \pm 0.4$ & $2.1 \pm 0.5$ & $2.2 \pm 0.5$ & $2.6 \pm 0.7$ & $9.133(2)$ & $<0.001$ \\
\hline
\end{tabular}

HEIAR: Hepatic echo intensity attenuation rate; dB: decibel, cm: centimetre; SCTT: Subcutaneous Tissue Thickness

According to the Chi-square test, Table 3 shows an association between SCTT and HEIAR with NAFLD. As grade I is considered initial stage of NAFLD, so that we adopted mean of the SCTT in grade I $(2.1 \mathrm{~cm})$ as a cut-off value to likely diagnose NAFLD where there were $65.4 \%(149 / 228)$ of subjects with SCTT measured $\geq 2.1 \mathrm{~cm}$ had NAFLD versus $34.6 \%$ (79/228) of subjects without NAFLD with sensitivity $52 \%$ and specificity $86 \%$. Among subjects with SCTT $<2.1 \mathrm{~cm}$, there were $21.5 \%(86 / 400)$ of subjects with NAFLD versus $78.5 \%$ (314/400) with no NAFLD.

Table 3 Chi-Square of HEIAR and SCTT with NAFLD

\begin{tabular}{lcccc}
\hline \multirow{2}{*}{ Variables } & \multicolumn{2}{c}{ NAFLD status } & \multirow{2}{*}{$\mathbf{X}^{2}(\mathbf{d f})$} & \multirow{2}{*}{$\boldsymbol{P}$-value } \\
\cline { 2 - 3 } HEIAR & Yes, $\boldsymbol{n}(\%)$ & No, $\boldsymbol{n}(\%)$ & $405.7(1)$ & $<0.001$ \\
$(\geq 1.7 \mathrm{~dB} / \mathrm{cm} . \mathrm{MHz}),(n=175)$ & $175(100.0)$ & $0(0.0)$ & & \\
$(<1.7 \mathrm{~dB} / \mathrm{cm} . \mathrm{MHz}),(n=453)$ & $60(13.2)$ & $393(86.7)$ & & \\
$\mathbf{S C T T}$ & & & $119.2(1)$ & $<0.001$ \\
$(\geq 2.1 \mathrm{~cm}),(n=228)$ & $149(65.4)$ & $79(34.6)$ & & \\
$(<2.1 \mathrm{~cm}),(n=400)$ & $86(21.5)$ & $314(78.5)$ & & \\
\hline
\end{tabular}


Sonographic Measurement of the Thickness of Subcutaneous Tissue and Hepatic Echo-Intensity Attenuation Rate in Non-alcoholic Fatty Liver Disease

Thus, Figure 3 illustrates the thinning of SCTT (less than $2.1 \mathrm{~cm}$ ) in subject with normal liver whereas Figure 4 illustrates the thickening of SCTT (more than $2.1 \mathrm{~cm}$ ) in a patient with NAFLD. Moreover, the mean HEIAR in grade I $(1.7 \mathrm{~dB} / \mathrm{cm}$. $\mathrm{MHz})$ has been depended for detecting NAFLD, where 175 out of $175(100.0 \%)$ subjects with HEIAR of $1.7 \mathrm{~dB} /$ $\mathrm{cm}$. $\mathrm{MHz}$ or above had NAFLD with sensitivity $59 \%$ and specificity $89 \%$. In contrast, only 60 out of $453(13.2 \%)$ subjects with NAFLD had HEIAR less than $1.7 \mathrm{~dB} / \mathrm{cm} . \mathrm{MHz}$.

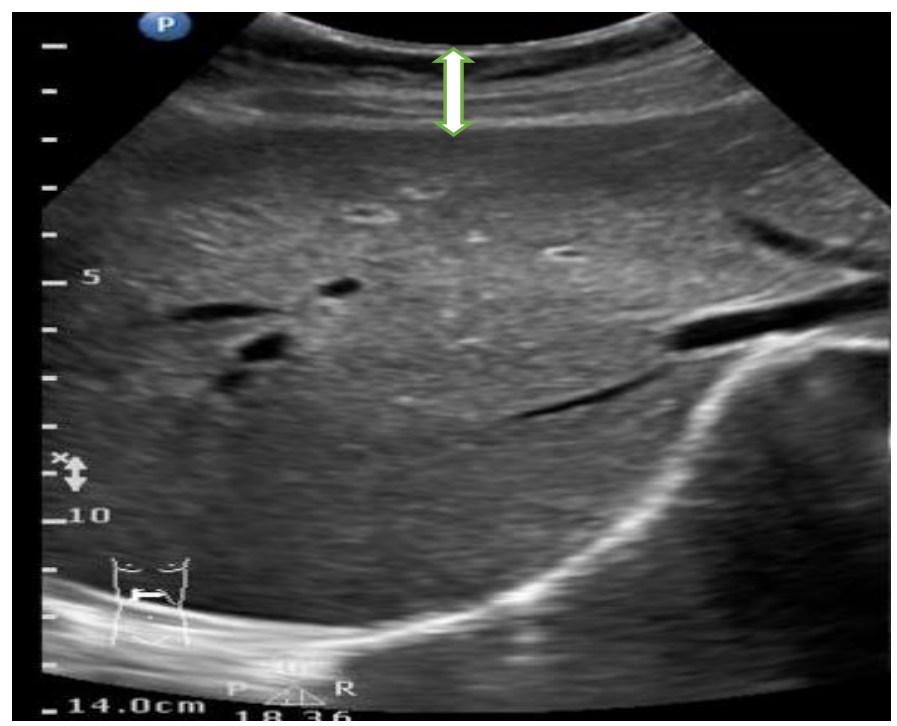

Figure 3 Normal liver sonography demonstrates a subcutaneous tissue thickness (arrow) of less than $2.1 \mathrm{~cm}$

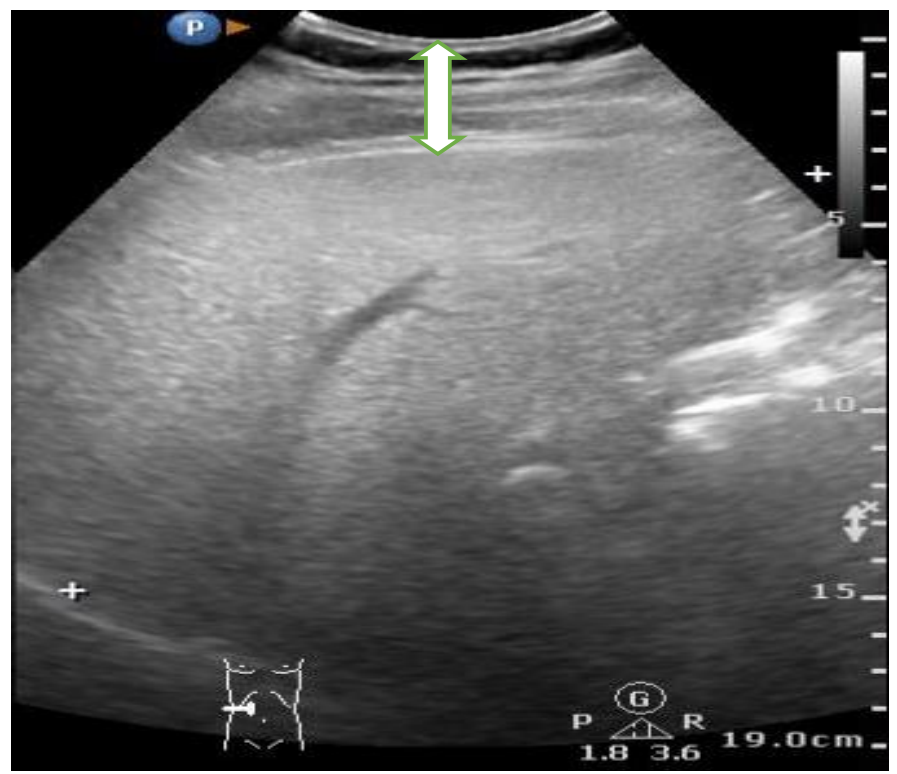

Figure 4 Sonography in of NAFLD patient demonstrates a subcutaneous tissue thickness (arrow) of more than $2.1 \mathrm{~cm}$ 


\section{DISCUSSION}

Although ultrasound is valuable to diagnose NAFLD, the liver biopsy is still considered as the reference tool for the detection, quantifying and grading of NAFLD as well as its ability to differentiate between NAFLD and nonalcoholic steatohepatitis (NASH) ${ }^{25,26}$. However, sampling error may occur because the fat infiltration is sometimes unequally distributed in hepatocytes ${ }^{27}$. Moreover, the biopsy is an invasive tool and has some risks which lead to serious complications. In this study, qualitative and quantitative ultrasound methods were used for assessment of fatty liver disease. The qualitative method describes the fatty liver disease based on increasing in reflectively of the liver parenchyma compared to the renal cortex ${ }^{28}$.

In contrary, the quantitative ultrasound method of HEIAR is an additional method that can be applied to the detection of NAFLD. In the present study, we present a method to quantify fatty infiltration in hepatocytes using ultrasound HEIAR. The ultrasound HEIAR is an objective, computer-calculated index. This idea is not new innovative but it is identical to concepts addressed in previously published studies with some amendments of practical points. Although Fujii et al. $(2002)^{29}$ and Itoh et al. (1988) $)^{30}$ indicated that sonographically measured the attenuation coefficient would be used for ultrasound quantitative assessment of the grading of fatty liver changes. The researchers needed specific systems to quantify the attenuation coefficient and these are hard to use in the factual clinical area. In our study, ultrasound evaluated the hepatic attenuation because it demonstrates a 256-level grayscale brightness on screens which is no highly accurate but could be useful. Although Webb et al. (2009) ${ }^{26}$ stated that the hepatorenal index was beneficial for quantification of hepatic steatosis, the various disease processes can, unfortunately, alter the echogenicity of the renal cortex. Moreover, the activated of time-gain compensation (TGC) activated as a default function using by most sonographers is considered a confounding factor to compensate for the echogenicity of deeper attenuated areas. Therefore, in the present study, we thwarted the activation of TGC because it is an inverse objective to measure the ultrasound attenuation.

In light of that, Sonographic quantitative assessment was used to quantify the mean of HEIAR among NAFLD grades as well as adoption these means in grade I as cut-off values to likely identify NAFLD. Our study reported a high difference of HEIAR among NAFLD grades. Importantly, mean HEIAR in grade I was $1.7 \mathrm{~dB} / \mathrm{cm} . \mathrm{MHz}$, thereby we found that the prevalence of NAFLD was $100 \%$ among patients who had HEIAR equal to or greater than $1.7 \mathrm{~dB} / \mathrm{cm} . \mathrm{MHz}$. Hence, sonographically measured HEIAR equal to or over than $1.7 \mathrm{~dB} / \mathrm{cm} . \mathrm{MHz}$ makes the diagnosis of NAFLD probably. Incompatible with our study, Zhang et al. (2012) ${ }^{31}$ confirmed that the difference of HEIAR between NAFLD and non-NAFLD was strongly significant. On equal importance, Kwon et al. (2013) 32 assessed cutoff values of the ultrasound attenuation index for estimation severe hepatic steatosis using two probes $4 \mathrm{MHz}$ and $8 \mathrm{MHz}$. The researchers reported that optimal ultrasound attenuation index cut-off value of 31.0 at $8 \mathrm{MHz}$ is beneficial to approach for non-invasive diagnosis of severe hepatic steatosis.

Furthermore, this study has also compared the means of SCTT among NAFLD grades where we depend on the mean in grade I as cut-off values to likely identify NAFLD. As expected, our results revealed a close correlation between SCTT and NAFLD grades. In addition, most of the healthy subjects had less than mean SCTT in grade I (less than 2.1 $\mathrm{cm})$. Our study is partially consistent with a study done by Riley \& Bruno (2005) ${ }^{33}$, which reported that the diagnosis of NAFLD among subjects with SCTT measuring sonographically less than $2.0 \mathrm{~cm}$ is unlikely. 
Sonographic Measurement of the Thickness of Subcutaneous Tissue and Hepatic Echo-Intensity Attenuation Rate in Non-alcoholic Fatty Liver Disease

\section{CONCLUSION}

Sonographically measured SCTT is not strongly approached to diagnose NAFLD. Nevertheless, HEIAR is a useful indicator for non-invasive quantitative assessment of NAFLD.

\section{ACKNOWLEDGMENTS}

The authors would like to acknowledge the help of Dr Norafida Bahari, a radiologist from the Department of Imaging, Faculty of Medicine and Health Sciences, Universiti Putra Malaysia. The authors are also thankful and grateful to all of the administrators and medical personnel who have willingly helped out with their abilities in Golden Horses Health Sanctuary, Klang Valley.

\section{CONFLICT OF INTEREST}

The authors declare that they have co competing interests in publishing this article.

\section{REFERENCES}

1. Li N, Zhang GW, Zhang JR et al. ( 2015). Nonalcoholic fatty liver disease is associated with progression of arterial stiffness. Nutrition, Metabolism and Cardiovascular Diseases 25 (2): $218-223$.

2. Neuschwander-Tetri BA, Caldwell SH. (2003). Nonalcoholic steatohepatitis: summary of an AASLD Single Topic Conference. Hepatology 37 (5): $1202-1209$

3. Chen C, Huang M, Yang J et al. (2006). Prevalence and risk factors of nonalcoholic fatty liver disease in an adult population of Taiwan: Metabolic significance of nonalcoholic fatty liver disease in nonobese adults. Journal of Clinical Gastroenterology 40 (8): 745 - 752.

4. Dai H, Chu L, Song S et al. (2009). Prevalence of and risk factors for fatty liver disease in a professional population of Wuhan, China. Public Health 123 (8): 545 - 548.

5. Fon TK, Rozman D. (2011). Nonalcoholic Fatty liver disease: focus on lipoprotein and lipid deregulation. Journal of Lipids 2011: 1 14. DOI: 10.1155/2011/783976.
6. Chitturi S, Farrell GC, Hashimoto E et al. (2007). Non alcoholic fatty liver disease in the Asia-Pacific region: Definitions and overview of proposed guidelines. Journal of Gastroenterology and Hepatology 22 (6): $778-787$.

7. Rampal L, Rampal S, Khor GL et al. (2007). A national study on the prevalence of obesity among 16,127 Malaysians. Asia Pacific Journal of clinical nutrition 16 (3): 561 - 566.

8. Oshibuchi M, Nishi F, Sato M et al. (1991). Frequency of abnormalities detected by abdominal ultrasound among Japanese adults. Journal of Gastroenterology and Hepatology 6 (2): 165 - 168.

9. Lai SW, Tan, CK, Ng KC. (2002). Epidemiology of fatty liver in a hospital-based study in Taiwan. Southern Medical Journal 95 (11): $1288-1293$.

10. Omagari K, Kadokawa Y, Masuda Jl et al. (2002). Fatty liver in non-alcoholic nonoverweight Japanese adults: Incidence and clinical characteristics. Journal of Gastroenterology and Hepatology 17 (10): $1098-1115$.

11. Shen L, Fan J, Shao $Y$ et al. (2003). Prevalence of nonalcoholic fatty liver among administrative officers in shanghai: An epidemiological survey. World J Gastroenterol 9 (5): 1106 - 1110.

12. Park SH, Jeon WK, Kim SH et al. (2006). Prevalence and risk factors of non-alcoholic fatty liver disease among Korean adults. Journal of Gastroenterology and Hepatology 21 (1): $138-143$.

13. Amarapurkar $\mathrm{D}$, Kamani $\mathrm{P}$, Patel $\mathrm{N}$ et al. (2007). Prevalence of non-alcoholic fatty liver disease: Population based study. Ann Hepatol 6 (3): $161-163$.

14. Fan JG, Saibara T, Chitturi S et al. (2007). What are the risk factors and settings for non-alcoholic fatty liver disease in AsiaPacific? Journal of Gastroenterology and Hepatology 22 (6): $794-800$.

15. Malik A, Cheah P, Hilmi IN et al. (2007). Nonalcoholic fatty liver disease in Malaysia: A demographic, anthropometric, metabolic and histological study. Journal of Digestive Diseases 8 (1): 58 - 64 .

16. Ahmed $\mathrm{MH}$, Barakat $\mathrm{S}$, Almobarak $\mathrm{AO}$. (2012). Nonalcoholic fatty liver disease and cardiovascular disease: has the time come for cardiologists to be hepatologists? Journal of Obesity 2012. 
17. Mehta SR, Thomas EL, Bell JD et al. (2008). Non-invasive means of measuring hepatic fat content. World Journal of Gastroenterology 14 (22): 3476 - 3483.

18. Hamad AA, Khalil AA, Connolly V, Ahmed MH. (2012). Relationship between non-alcoholic fatty liver disease and kidney function: A communication between two organs that needs further exploration. Arab Journal of Gastroenterology 13 (4): 161 - 165.

19. Targher G, Marra F, Marchesini G. (2008). Increased risk of cardiovascular disease in non-alcoholic fatty liver disease: causal effect or epiphenomenon? Diabetologia 51 (11): 1947 - 1953.

20. Lindbäck SM, Gabbert C, Johnson BL et al. (2010). Pediatric nonalcoholic fatty liver disease: A comprehensive review. Advances in Pediatrics 57 (1): 85 - 140.

21. Ma X, Holalkere $\mathrm{N}$, Mino-Kenudson $\mathrm{M}$ et al. (2009). Imaging-based quantification of hepatic fat: Methods and clinical applications. Radiographic 29 (5): 1253 - 1277.

22. Singh D, Das CJ, Baruah MP. (2013) Imaging of non alcoholic fatty liver disease: $A$ road less travelled. Indian Journal of Endocrinology and Metabolism 17(6): 990 - 995.

23. Saadeh S, Younossi ZM, Remer EM et al. (2002). The utility of radiological imaging in nonalcoholic fatty liver disease. Gastroenterology 123 (3): 745 - 750.

24. Williamson R, Perry E, Glancy S et al. (2011). The use of ultrasound to diagnose hepatic steatosis in type 2 diabetes: Intra-and interobserver variability and comparison with magnetic resonance spectroscopy. Clinical Radiology 66 (5): 434 - 439.

25. El-Koofy N, El-Karaksy $\mathrm{H}$, El-Akel $\mathrm{W}$ et al. (2012). Ultrasonography as a non-invasive tool for detection of nonalcoholic fatty liver disease in overweight/obese Egyptian children. European Journal of Radiology 81 (11): $3120-3123$.
26. Webb $M$, Yeshua $H$, Zelber-Sagi $S$ et al. (2009). Diagnostic value of a computerized hepatorenal index for sonographic quantification of liver steatosis. American Journal of Roentgenology 192 (4): 909 - 914.

27. Wai CT, Tan LHC, Kaur M et al. (2002). Pitfalls in interpreting liver biopsy results: the story of the blind men and the elephant. Liver transplantation 8 (12): $1200-1201$.

28. Chen S, Liu C, Li S et al. (2008). Effects of therapeutic lifestyle program on ultrasounddiagnosed nonalcoholic fatty liver disease. Journal of the Chinese Medical Association 71 (11), $551-558$.

29. Fujii $\mathrm{Y}$, Taniguchi $\mathrm{N}$, Itoh $\mathrm{K}$ et al. (2002). A new method for attenuation coefficient measurement in the liver Comparison with the spectral shift central frequency method. Journal of Ultrasound in Medicine 21 (7): 783 - 788.

30. Itoh K, Yasuda Y, Suzuki O et al. (1988). Studies on frequency-dependent attenuation in the normal liver and spleen and in liver diseases, using the spectral-shift zero-crossing method. Journal of Clinical Ultrasound (168): $553-562$.

31. Zhang B, Ding F, Chen T et al. (2014). Ultrasound hepatic/renal ratio and hepatic attenuation rate for quantifying liver fat content. World Journal of Gastroenterology 20 (47): $17985-17992$.

32. Kwon HJ, Kim KW, Lee SJ et al. (2013). Value of the ultrasound attenuation index for noninvasive quantitative estimation of hepatic steatosis. Journal of Ultrasound in Medicine 32 (2): 229 - 235.

33. Riley TR, Bruno MA. (2005). Sonographic measurement of the thickness of subcutaneous tissues in nonalcoholic fatty liver disease versus other chronic liver diseases. Journal of Clinical Ultrasound 33 (9): $439-441$. 